\title{
Mitochondria: A Key Player in Stem Cell Fate
}

\author{
Nadra Sadaf ${ }^{1}$, Jawaid Ahsan ${ }^{1}$, Saif Ahmad ${ }^{2}$, Rizwanul Haque ${ }^{1, ~ *}$ \\ ${ }^{1}$ Centre for Biological Sciences (Biotechnology), Central University of South Bihar, Patna, India \\ ${ }^{2}$ Dept of Biological Sciences, College of Science and Arts, King Abdulaziz University, Rabegh, Kingdom of Saudi Arabia
}

\author{
Email addresses: \\ rhaque@cub.ac.in (R. Haque)
}

\section{To cite this article:}

Nadra Sadaf, Saif Ahmad, Rizwanul Haque. Mitochondria: A Key Player in Stem Cell Fate. Cell Biology. Special Issue: Mitochondria: Implications in Human Health and Diseases. Vol. 3, No. 2-1, 2015, pp. 31-37. doi: 10.11648/j.cb.s.2015030201.15

\begin{abstract}
Mitochondria are highly dynamic organelles that undergo cycles of fusion and fission important for their function, maintenance, and quality control as well as direct or indirect role in different types of stem cells fate decisions. Stem cells have potential for numerous biomedical applications; however the major bottle neck in stem cell field is the stem cell differentiation and maintenance of stemness of stem cells. The regulatory factor involved in stem cell fate decision and stem cell development is not clear. Recent report suggests that mitochondria also play a role in maintenance of pluripotency and cell fate decision. This review mainly cover the role of mitochondria, reactive oxygen species and change in mitochondria structure and functions during cell fate decision in different types of major group of stem cells. This article is a part of special issue on Mitochondria.
\end{abstract}

Keywords: Pluripotent Stem Cells (PSCs), Embryonic Stem Cells (ESCs), Induced Pluripotent Stem Cells (iPSCs), Adult Stem Cell (ASCs), Cancer Stem Cells (CSCs), Mitochondria, Reactive Oxygen Species (ROS)

\section{Introduction}

Mitochondria are compartmentalized endosymbiotic bacterial origin organelles present in all eukaryotic cells except in red blood cells and in some protozoans. They play an important role in many cellular functions including pyrimidine biosynthesis, ion homeostasis [1], fatty acid oxidation, apoptosis [2] and cell signalling [3]. One of the mitochondrion's key functions is the generation of adenosine triphosphate (ATP), the cell's major source of energy. Mitochondria are semiautonomous organelles having its own $16.6 \mathrm{~kb}$ long DNA in humans and it is extremely compact contains only two noncoding regions and no introns between adjacent genes. mtDNA encodes 22 transfer RNAs (tRNAs) and two ribosomal RNAs (rRNAs), which are required for mitochondrial protein synthesis [4,5]. Mitochondria are also the major sources of endogenous reactive oxygen species (ROS), which are by-products of ATP production through oxidative phosphorylation. Although high levels of ROS may cause protein carbonylation, lipid peroxidation, and DNA damage and have deleterious effects for the cells [6]. Mitochondria have been closely linked to cell fate determination and development, and several reports have demonstrated important roles of mitochondria in stem cells $[7,8,9]$. Mitochondria are an energy powerhouse responsible for ATP production, play a pivotal role in supplying the energy required during cell multiplication and cell lineages differentiation. Characterization of different cell types based on mitochondrial properties and localization indicates that the mitochondrial phenotype is an important consideration in the analysis of differentiated hESC progeny [10]. Several studies have demonstrated that there is a strong connection between mitochondrial function and stemness/pluripotency $[11,12,13,14]$. Mitochondria are an energy powerhouse of all cells including stem cell and various studies proved that mitochondria play an important role in stem cell differentiation and self-renewal.

\section{Mitochondrial ROS in Stem Cell}

ROS are implicated at many distinct levels of biological processes, from gene expression and protein translation to protein-protein interactions. ROS may function as a rheostat to coordinate various cellular processes and adjust cellular activity to the available bioenergetic sources by propagating signals from one tissue to the next, and translating environmental cues into cellular responses [15]. ROS are organic and inorganic molecules that have an odd number of electrons in their outer valence shell. $\mathrm{H}_{2} \mathrm{O}_{2}$ is thought to be the main ROS species involved in intracellular signalling due to its longer half-life and ability to diffuse easily through membranes relative to other types of ROS [16].The 
mitochondrial electron transport chain is the main source of ROS within cell and it is found that $0.1-0.2 \%$ of $\mathrm{O}_{2}$ consumed by mitochondria is thought to form ROS through the premature electron flow to $\mathrm{O}_{2}$, mainly through electron transport chain complexes I and III [17]. ROS can signal directly to proteins via amino acid oxidation the most common reaction is oxidation of cysteine residues. ROS signalling to amino acids can cause functional changes in proteins and thus these types of modifications have established ROS as crucial regulators of cellular signalling such as influences their function, stability, subcellular localization, interactions with other proteins and other crucial processes like stem cell fate regulation [Fig. 1].

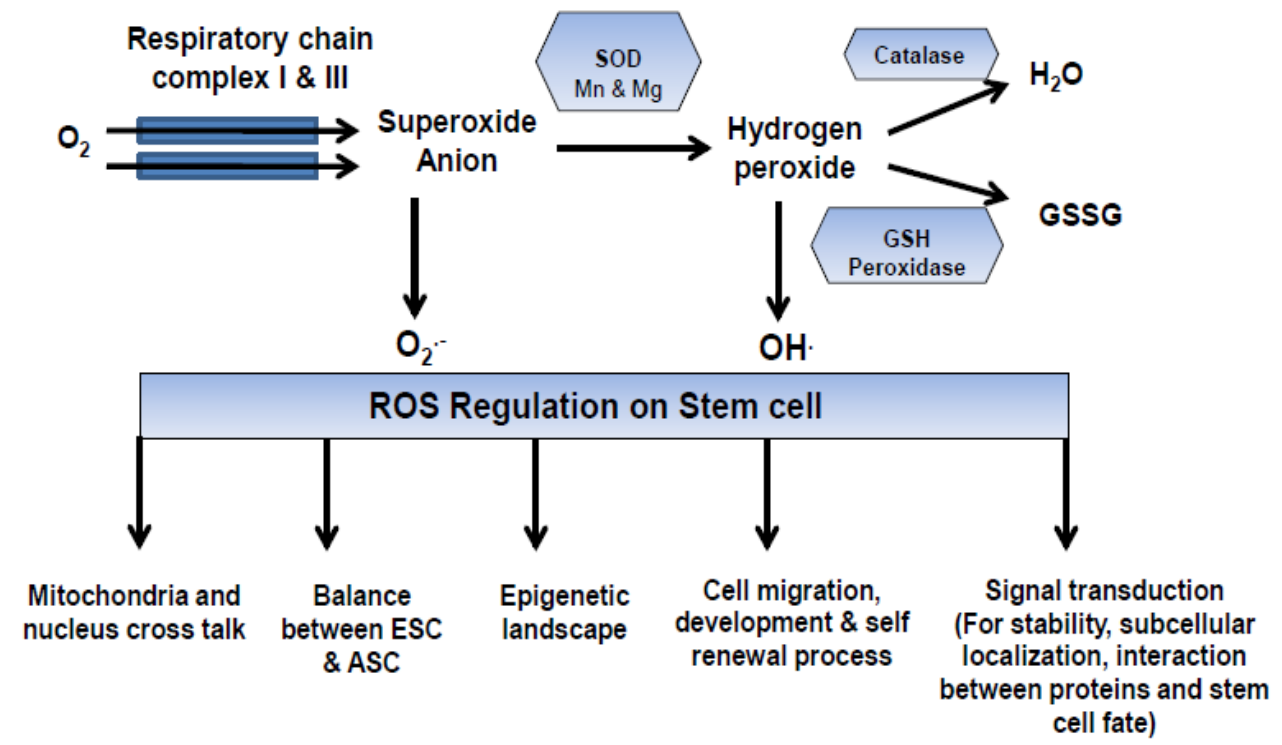

Figure 1. Regulatory role of ROS in different signaling that affect stem cells. ROS generated through respiration involve in cross talk between mitochondria \& nucleus, cell fate, epigenetic changes, cell migration, self-renewal and signal transduction pathways (SOD; superoxide dismutase, GSSG; Glutathione disulfide, GSH; Glutathione).

An appropriate balance between self-renewal and differentiation is crucial for stem cell function during both early development and tissue homeostasis throughout life. Recent studies suggest that the balance between pluripotent embryonic and adult stem cell is partly regulated by ROS and metabolism that mediate the cellular redox state [18]. Oxidative stress particularly ROS influences stem cell migration, development, and self-renewal as well as their cell cycle status [19]. ROS play role in physiological regulation of crucial developmental processes, such as the emergence of embryonic blood stem cells or differentiation of embryonic cardiomyocytes [20]. The Redox-mediated mitochondria and nucleus crosstalk could explain the coordination of cellular metabolism with chromatin remodeling, gene expression, cell cycling, DNA repair and cell differentiation. ROS might also function to alter the epigenetic landscape, which plays a role in regulating stem cell fate $[21,22]$. ROS can be considered as signalling molecules that take part in the crosstalk between metabolism and stem cell fate decisions.

\subsection{Role of Mitochondria in ESCs}

Embryonic stem (ES) cells are pluripotent stem cell derived from the pre-implantation blastocyst, a hollow sphere of cells containing an outer layer of trophoblast cells which give rise to the placenta and the inner cell mass (ICM), from which ES cells are derived. Cells of the ICM ultimately form the embryo and therefore have the capacity to form all the tissues in the body.ES cells retain the character of embryo founder cells, even after prolonged culture and extensive manipulation. ES cells closely resemble EC cells in morphology, growth behaviour, and marker expression. A key property of ES cells is that they maintain euploid karyotype. Membrane proteins are the most important marker type in recognizing ESC. Some Stage Specific Embryonic Antigens such as lacto- and globo-series glycolipids SSEA-1, SSEA-3, and SSEA-4 are involved in controlling cell surface interactions during development. The $\mathrm{CD}$ antigens associated with pluripotent hES cells are CD9, CD24, CD5, CD31\& CD133 and few specific markers are TRA-1-60, TRA-1-81, Frizzled5\& SCF or c-Kit ligand. Pluripotent stem cells (PSCs) includes embryonic stem cells (ESCs) and induced pluripotent stem cells (iPSCs) that have an unlimited capacity for self-renewal and can differentiate into any cell type in our bodies. PSCs have a short G1 phase of the cell cycle as compared to somatic cell that limits the growth and differentiation potential of PSC [23,24]. Embryonic stem cells (ESCs) contain relatively few spherical and immature mitochondria that is heavily depend on anaerobic glycolysis for energy production [11,13] that support cell proliferation of PSCs. ESC mitochondria have a lower respiratory capacity but a higher mitochondrial membrane potential, an important component of the proton motive force [14]. Although glycolysis is less efficient in terms of energy production, it produces energy at a faster rate with lower ROS generation, which is important for maintaining pluripotency under hypoxic conditions so, stimulation of glycolysis by hypoxia or inhibition of 
mitochondrial respiration promotes pluripotency. PSCs contain functionally immature mitochondria with a globular shape, poorly developed cristae, and perinuclear localization [13]. This indicates that less active and immature mitochondrial state help in the maintenance of pluripotency. The NANOG, POU5F1 (OCT4) and SOX2 are critical pluripotency genes involved in cell differentiation potential and increases the pluripotency state that was observed by attenuating mitochondrial function in undifferentiated human ESCs by increasing the mRNA levels of these genes. Human ESCs (hESCs) exhibit improved self-renewal and pluripotency in $3 \%-5 \%$ (physiological) lower O2 relative to atmospheric O2. Recently published papers suggest that when mitochondrial mass is measured by mitochondrial proteins and mtDNA copy number the ratio between these cellular parameters is similar in ESCs and differentiated cells [25,14]. The mtDNA haplotypes in ESCs is observed due to epigenetic alterations of genes;mtDNA haplotypes influence not only chromosomal gene expression but also cell fate determination upon differentiation. Amino acids and fatty acids are also required for PSC self-renewal and differentiation. Ptpmt1, a mitochondrial Pten-like phosphatidylinositol phosphate (PIP) phosphatase plays a critical role in early embryogenesis of ESCs and depletion of Ptpmt1 impairs ES cell maintenance and causes stem cell-specific mitochondrial stress that trigger cell cycle checkpoints [26]. Metabolomic data of purine nucleotides in ESCs and somatic cell indicates that purine nucleotides are differentially present between these cells [27]. Epigenetic landscape is also one of the factor that regulate stem cell by the methylation of CpG islands in DNA through SAM (s-adenosyl methionine) and it is highly upregulated pathway in embryonic stem cells [28]. Mandal et al., in 2011 found that differentiation of stem cell results in the generation of an extensive network of branched mitochondria that causes change in mitochondrial morphology and the loss of OCT4 and NANOG mRNA, finally the loss of pluripotency state [29]. It has also been found that mitochondrial fission is involve in maintenance of pluripotency by the gene knockdown of the mitochondrial protein Gfer (growth factor erv1-like) in mouse ESCs that leads to decreased levels of pluripotent markers (NANOG, OCT4, \&SSEA) through the regulation of dynamin-related protein 1 (Drp1), an important mitochondrial fission GTPase. On differentiation pleuripotent stem cell mitochondria undergo significant changes like mtDNA copy number is elevated, and mitochondrial morphology displays a structurally mature state with dense matrix, complex cristae and dispersed cytoplasmic localization. Above reported studies suggest that mitochondrial function and dynamics play an important role in the expression of pleuripotent specific genes and influence different other parameters change in membrane potential, glycolysis, amino acids and fatty acids production to maintain pluripotency [Fig 2].

\subsection{Role of Mitochondria in iPSCs}

Induced pluripotent stem cells (iPSCs) are reversibly "transformed" somatic cells and are derived by introducing a specific set of pluripotency-associated genes or "reprogramming factors" into an adult cell. Induced pleuripotent stem cells (iPSCs) is a type of pluripotent stem cells that can be generated in the laboratory by over expressing ESC specific genes such as Oct4 (Pou5f1), Nanog, Sox2, $\mathrm{cMyc}$, and Klf4 in mature adult cells and thus reprogramming them to an ESC like state [30,31]. Reprogramming of somatic cells into a pluripotent state is successfully established by Takahashi et al. in 2006, through the induction of four pluripotent specific transcription factors [32]. Mitochondrial coordinated dynamics are vital in stem cell for the mitochondrial metabolism, energy production, ROS production, calcium signalling, and apoptosis [33]. Unbalanced fusion or fission leads to impairment of mitochondrial dynamics, which is being increasingly implicated in human diseases, such as neurodegeneration and muscle atrophy [34]. Mitochondrial fission and fusion events that regulate mitochondrial distribution have also play important roles in the reprogramming process. Reprogramming induces structural and functional remodeling of parental mitochondria such as reduction in mtDNA copy number and changes in the structure and morphology of mitochondria to a functionally immature state [Fig. 2]. After reprogramming of adult cell there is a change in energy production from somatic oxidative phosphorylation to glycolysis that is achieved through transcriptional and epigenetic regulation $[11,13,35]$. It has been also reported that switching of glycolysis in induced pluripotent cell from oxidative phosphorylation is necessary to reprogram somatic cells to pluripotency state [14]. Cells with higher mitochondrial membrane potential, express glycolytic genes (Glut1, Hxk2, Pfkm, and Ldha) significantly within first week of reprogramming,whereas expressions of pluripotent genes (Fgf4, Nanog, Oct4, and Sox2) remained at low levels. iPSCs and ESCs have similar glycolytic metabolism but shows differences at the levels of unsaturated fatty acids and S-adenosyl methionine that may play important roles during reprogramming [27]. Acetyl-CoA carboxylase and fatty acid synthase are highly expressed in iPSCs and inhibition of this enzymes leads to in reprogramming efficiency of somatic cells [36]. Phospholipids a potent signalling molecule including phosphatidylinositol 4,5-bisphosphate play an important role in mitochondrial membrane processes [37]. After reprogramming, cell start behaving like PSC by inducing pluripotent specific genes such as Oct4 or Nanog and induces structural and functional remodelling in mitochondria for other PSC related functional changes.

\subsection{Role of Mitochondria in ASCs}

Adult stem cells also known as somatic stem cells are undifferentiated cells, found throughout the body after development, which multiply by cell division to replenish dying cells and regenerate damaged tissues. Embryonic stem cells (ESCs) and adult stem cells are naturally occurring stem cells [38]. Mitochondrial staining is one of the common techniques that are used as an adult stem cell indicator due to the difference in function and dynamics of mitochondria in 
different types of stem cells. The multipotent adult stem cell derived from adult tissue which can differentiate into a limited number of cell types of their own lineage. Few examples of human adult stem cells are hematopoietic stem cells or mesenchymal stem cells (MSCs) that are limited in their self-renewal capacity and the number of target lineages in which they can differentiate. Hematopoietic stem cells are located in the endosteal niche or in the vascular niche within the bone marrow [39]. HSCs contains relatively immature mitochondria that suggest HSCs contain mitochondria with low levels of activity such as lower respiratory rate and a low mitochondrial membrane potential as compared to downstream progenitor cells [40]. MSCs are the non-hematopoietic progenitor cells found in various adult tissues have rapid growth in vitro while maintaining their differentiation potential. There is difference in mitochondrial membrane potential between ESCs and HSCs that shows the proliferative and 'primed to differentiate' nature of ESCs as compared to HSCs which are mostly quiescent in nature [Fig 2].

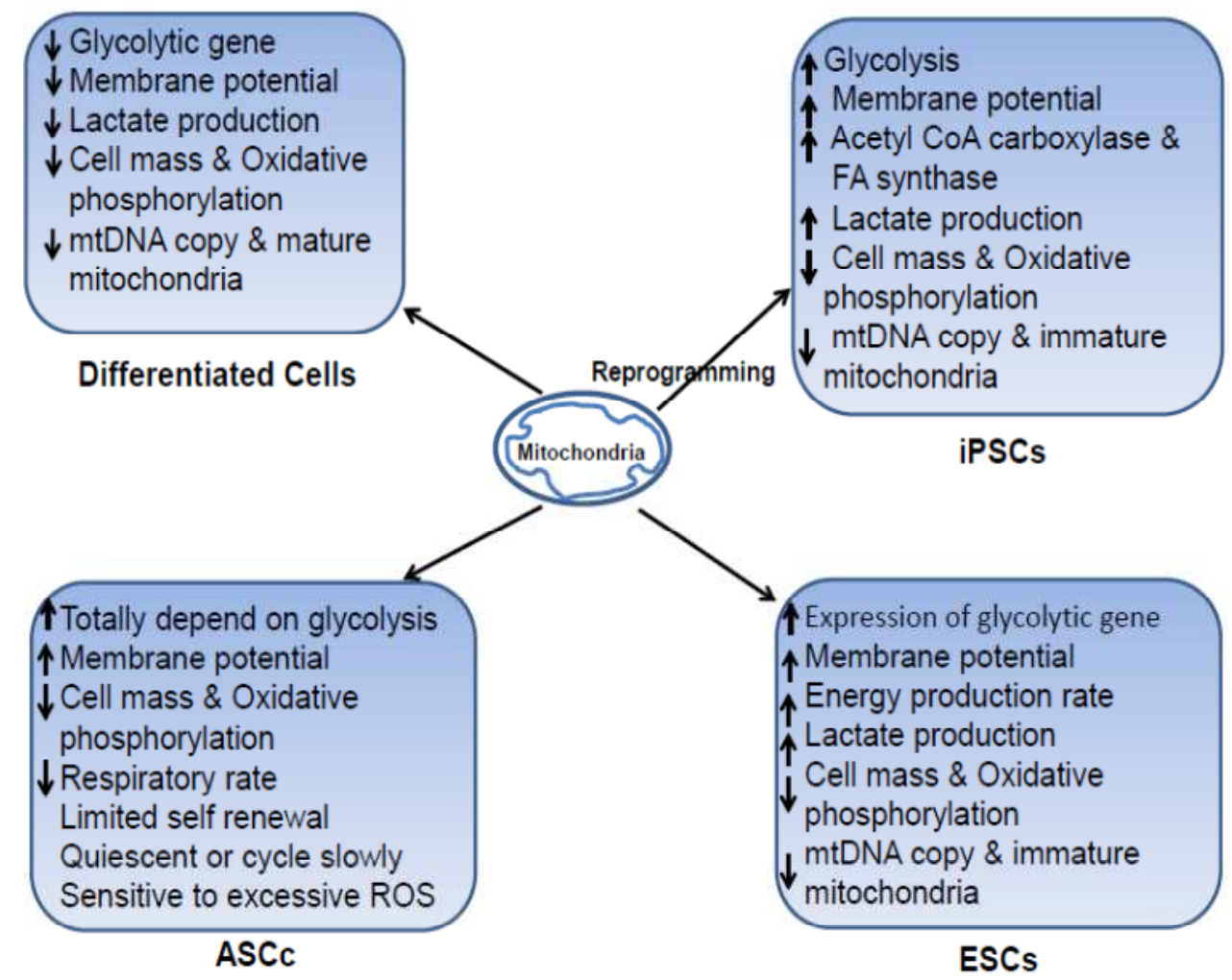

Figure 2. Role of mitochondria in different types of stem cells. Mitochondria and its function in different types of stem cells are more or less same (adult stem cell fully depends on glycolysis) but vary drastically in differentiated cells as compare to stem cells.

It is therefore conceivable that it is the mitochondrial membrane potential and not the type of metabolism that is indicative of the degree to which stem cells are primed to differentiate. Increased level of ROS causes the elevated expression of tumor suppressors (p16Ink4a and p19Arf) that activate p38MAPK, leading to the HSC compartment loss [41]. SAM (s-adenosyl methionine) and TET (ten eleven translocase) enzymes are crucial factors in epigenetic landscape that regulate hematopoietic stem cells. Adult stem cells are limited in their self-renewal capacity as well as number of target lineages in which they differentiate as compared to ESCs and epigenetic landscape and ROS are involve in regulating hematopoietic stem cells.

\subsection{Mitochondria in Cancer Stem Cell}

mtDNA is highly vulnerable to mutations due to the high levels of reactive oxygen species (ROS) and reduced antioxidant defenses in mitochondria. The possible presence of stem cell populations in tumors has many implications for the diagnosis and treatment of cancers $[42,43]$. Cancer stem cells (CSCs) are believed as the initiators of the occurrence, development and recurrence of malignant tumors. ES cells and cancer stem cells share several traits, including unlimited self-renewal capabilities and the ability to generate a diverse range of other cell types but tumorigenic potential and unique profiles of surface markers specifically present in cancer stem cell $[35,44]$. Cancer cell mitochondria are structurally and functionally different from their normal counterparts. The mitochondrial phenotype of CSCs remains unknown and shows difference in mitochondrial membrane potential and reactive oxygen species [45]. Percentage of cells with aperinuclear mitochondrial arrangement might serve as an indicator of the stem cells [46] and possess a higher membrane potential than normal epithelial cells at least above $60 \mathrm{mV}$ [47]. Schieke et al.,[48] detected that the cells with higher membrane potential were more prone to continue dividing and form tumors, while lower membrane potential cells were more efficient indifferentiating into other cell types. The CSC 
biomarker CD133 expression could be detected in the higher membrane potential cells, while it is nearly absent in the other cells. Mitochondrial uncoupling proteins (UCPs) may be related to cancer initiation because $\mathrm{UCP} 2^{-/-}$cells displayed enhanced colony formation and hypoxia resistance, Derdak et al.,[49] observed that $\mathrm{UCP} 2^{-/-}$mice developed more colon tumors than $\mathrm{UCP} 2^{+/+}$. Cancer cells produce more ROS than normal cells and are involved in each stage of cancer development, including the initiation, promotion, and progression [50]. CSCs might have a high antioxidant capacity to keep cellular ROS at a moderate level, not like bulk cancer cells and maintain both stemness and cancer forming capabilities. Mitochondrial parameters observed in cancer stem cells (CSCs) including mitochondrial mass, mitochondrial morphology, mtDNA copy number, and oxygen consumption are similar to those observed in iPSCs.

\section{Conclusions}

Mitochondria are one of the powerful intracellular organelle that primarily function to generate ATP and actively involved in various regulatory functions such as in stem cell fate through the change in its appearance and activity. In order to meet different demands of distinct cell types and tissues, cells modulate mitochondrial function through biogenesis and degradation as well as dynamic fusion and fission events. Mitochondria are also the main producer of ROS that is involved in stem cell cellular redox state maintenance, migration, development, and self-renewal as well as their cell cycle status. ROS also alter the epigenetic landscape that have role in regulating stem cell fate but variations in ROS content may have profound effects on stem cell fate. Change in membrane potential, glycolysis, amino acids and fatty acids production is important in maintaining pluripotency state in stem cell. Reprogramming of somatic cells shows the change in mitochondria to produce induced pluripotent cell. iPSCs and CSCs have some common metabolic features that may help in their further characterization and study. Mitochondrial genes and proteins can be modulated for the maintenance of pluripotency and increasing the efficiency of reprogramming of somatic cells into pluripotent cells. Identifying mitochondrial specific genes or proteins in cancer stem cells may open new avenues for targeting cancer stem cells to cure one of the most ancient untreatable diseases like cancer.

\section{Acknowledgements}

This work was supported by Central University of South Bihar, Patna, India and Deanship of Scientific Research (DSR), King Abdulaziz University, Jeddah, Saudi Arabia.

\section{References}

[1] J. Jacobson and M.R. Duchen, "Interplay between mitochondria and cellular calcium signaling" Mol. Cell. Biochem. Vol. 256-257(1-2), pp. 209-218, 2004.
[2] N.N. Danial and S.J. Korsmeyer "Cell death: critical control points". Cell vol. 116, pp. 205-219, 2004.

[3] S.D. Dyall, M.T. Brown and P.J. Johnson, "Ancient invasions: from endosymbionts to organelles". Science vol. 304, pp. 253-257, 2004.

[4] G. Attardi, "Animal mitochondrial DNA: An extreme example of genetic economy". International Review of Cytology, vol. 93, pp. 93-145, 1985.

[5] S. Anderson, A. T. Bankier, and B. G. Barrell, "Sequence and organization of the human mitochondrial genome". Nature, vol. 290, pp. 457-465, 1981.

[6] L.A. Sena, and N.S. Chandel, "Physiological roles of mitochondrial reactive oxygen species". Mol. Cell, vol. 48, pp.158-167, 2012.

[7] L. Thomas and B. Bavistar, "Mitochondria in stem cells". Mitochondrion, vol. 7(5), pp. 289-296, 2007.

[8] C.D. Folmes, P.P. Dzeja, T.J. Nelson, and A. Terzic, "Metabolic plasticity in stem cell homeostasis and differentiation". Cell Stem Cell vol. 11, pp. 596-606, 2012.

[9] V.A. Rafalski, E. Mancini, and A. Brunet, "Energy metabolism and energy-sensing pathways in mammalian embryonic and adult stem cell fate". J. Cell Sci., vol. 125, pp. 5597-5608, 2012.

[10] F. Hattori, H. Chen, H. Yamashita, S. Tohyama and Y.S. Satoh, "Nongenetic method for purifying stem cell-derived cardiomyocytes". Nat Methods, vol. 7, pp. 61-66, 2010.

[11] C.D. Folmes, T.J. Nelson, A. Martinez-Fernandez, D.K. Arrell, J.Z. Lindor, P.P. Dzeja, Y. Ikeda, C. Perez-Terzic, and A. Terzic, "Somatic oxidative bioenergetics transitions into pluripotency-dependent glycolysis to facilitate nuclear reprogramming”. Cell Metab., vol. 14, pp. 264-271, 2011.

[12] L. Lapasset, O. Milhavet, A. Prieur, E. Besnard, A. Babled, N. Ai"t-Hamou, J. Leschik, F. Pellestor, , J.M. Ramirez, , J. De Vos, "Rejuvenating senescent and centenarian human cells by reprogramming through the pluripotent state". Genes Dev., vol. 25, pp. 2248-2253, 2011.

[13] A. Prigione, B. Fauler, R. Lurz, H. Lehrach, J. Adjaye, "The senescencerelated mitochondrial/oxidative stress pathway is repressed in human induced pluripotent stem cells". Stem Cells, vol. 28, pp. 721-733, 2010.

[14] J. Zhang, I. Khvorostov, J.S. Hong, Y. Oktay, L. Vergnes, E. Nuebel, P.N. Wahjudi, K. Setoguchi, G. Wang and A. Do, "UCP2 regulates energy metabolism and differentiation potential of human pluripotent stem cells". EMBO J., vol. 30, pp. 4860-4873, 2011.

[15] K. Wang, T. Zhang, Q. Dong, E. C. Nice and C.Huang, Redox homeostasis: the linchpin in stem cell self-renewal and differentiation. Cell Death Dis., vol. 4(3), article e537, 2013.

[16] G. C. Brown and V. Borutaite, "There is no evidence that mitochondria are the main source of reactive oxygen species in mammalian cells," Mitochondrion, vol. 12(1), pp. 1-4, 2012.

[17] S. Pervaiz, R. Taneja, and S. Ghaffari, "Oxidative stress regulation of stem and progenitor cells," Antioxidants and Redox Signaling, vol. 11(11), pp. 2777-2789, 2009. 
[18] L. B. Carolina, "Stem cells and the impact of ROS signaling". Development, vol. 141, pp. 4206-4218, 2014.

[19] A. Ludin, "Reactive Oxygen Species Regulate Hematopoietic Stem Cell Self-Renewal, Migration and Development, As Well As Their Bone Marrow Microenvironment". ANTIOXIDANTS \& REDOX SIGNALING Vol. 21(11), pp. 1605-1619, 2014.

[20] J.R. Hom, R.A. Quintanilla, D.L. Hoffman, K.L. de Mesy Bentley, J.D. Molkentin, , S.S. Sheu, and G.A. Porter, "The permeability transition pore controls cardiac mitochondrial maturation and myocyte differentiation". Dev.Cell, 21, pp. 469-478, 2011.

[21] T. Ishii and G. E. Mann, "Redox status in mammalian cells and stem cells during culture in vitro: critical roles of $\mathrm{Nrf} 2$ and cystine transporter activity in the maintenance of redox balance," Redox Biology, vol. 2, pp. 786-794, 2014.

[22] A. Valle-Prieto and P. A. Conget, "Human mesenchymal stem cells efficiently manage oxidative stress," Stem Cells and Development, vol.19, no. 12, pp. 1885-1893, 2010.

[23] A.M. Singh, and S. Dalton, "The cell cycle and Myc intersect with mechanisms that regulate pluripotency and reprogramming”. Cell Stem Cell, vol. 5, pp. 141-149, 2009.

[24] Y. Wang, S. Baskerville, A. Shenoy, J.E. Babiarz, L. Baehner, and R. Blelloch, "Embryonic stem cell-specific microRNAs regulate the G1-S transition and promote rapid proliferation". Nat. Genet., vol. 40, pp. 1478-1483, 2008.

[25] M.J. Birket, A.L. Orr, A.A. Gerencser, D.T. Madden, C. Vitelli, A. Swistowski, M.D. Brand and X. Zeng, "A reduction in ATP demand and mitochondrial activity with neural differentiation of human embryonic stem cells". J. Cell Sci., vol. 124, pp. $348-358,2011$

[26] J.Shen, X.Liu , W.M.Yu , J.Liu, M.G. Nibbelink, C.Guo, T. Finkel and C.K. Qu, "A Critical Role of Mitochondrial Phosphatase Ptpmt1 in Embryogenesis Reveals a Mitochondrial Metabolic Stress-Induced Differentiation Checkpoint in Embryonic Stem Cells". Mol. Cell. Biol., vol. 31(24), pp. 4902-4916, 2011.

[27] A.D. Panopoulos, O. Yanes, S. Ruiz, Y.S. Kida, D. Diep, R. Tautenhahn, A. Herrerias, E.M. Batchelder, N. Plongthongkum, M. Lutz, W.T. Berggren, K. Zhang, R.M. Evans, G. Siuzdak and J.C. Belmonte, "The metabolome of induced pluripotent stem cells reveals metabolic changes occurring in somatic cell reprogramming”. Cell Res., vol. 22, pp. 168-177, 2011.

[28] J. Wang, P. Alexander, L.Wu, R. Hammer, O. Cleaver and S.L. McKnight, "Dependence of mouse embryonic stem cells on threonine catabolism”. Science vol. 325, pp. 435-439, 2009.

[29] S. Mandal, A.G. Lindgren, A.S. Srivastava, A.T. Clark, and U. Banerjee, "Mitochondrial function controls proliferation and early differentiation potential of embryonic stem cells". Stem Cells, vol. 29, pp. 486-495, 2011.

[30] S. Yamanaka, "A fresh look at iPS cells". Cell, vol. 137, pp. 13-17, 2009.

[31] S. Nishikawa, R.A. Goldstein, C.R. Nierras, "The promise of human induced pluripotent stem cells for research and therapy". Nat Rev Mol Cell Biol., vol. 9, pp. 725-729, 2008.

[32] K. Takahashi, S. Yamanaka, "Induction of Pluripotent Stem Cells from Mouse Embryonic and Adult Fibroblast Cultures by
Defined Factors.” Cell vol. 126(4), pp. 663-676, 2006.

[33] D.C. Chan, "Mitochondria: dynamic organelles in disease, aging, and development". Cell, vol. 125, pp. 1241-1252, 2006.

[34] H. Chen, "Mitochondrial fusion is required for mtDNA stability in skeletal muscle and tolerance of mtDNA mutations". Cell, vol. 14,1pp. 280-289, 2010.

[35] S. Varum, A.S. Rodrigues, M.B. Moura, O. Momcilovic, C.A. Easley, "Energy metabolism in human pluripotent stem cells and their differentiated counterparts"., vol. 6(6), pp. 1-16, 2011.

[36] A. Vazquez-Martin, B. Corominas-Faja, S. Cufi, L. Vellon, C. Oliveras-Ferraros, O.J. Menendez, J. Joven, R. Lupu and J.A. Menendez, "The mitochondrial H(+)-ATP synthase and the lipogenic switch: new core components of metabolic reprogramming in induced pluripotent stem (iPS) cells". Cell Cycle, vol. 12, pp. 207-218, 2013.

[37] C. Osman, D.R. Voelker and T. Langer, "Making heads or tails of phospholipids in mitochondria". J. Cell Biol., vol. 192, pp. 7-16, 2011.

[38] K.W. Orford and D.T. Scadden, "Deconstructing stem cell selfrenewal: genetic insights into cellcycle regulation". Nat Rev Genet., vol. 9, pp. 115-128, 2008.

[39] S.J. Morrison, D.T.Scadden, "The bone marrow niche for haematopoietic stem cells”. Nature, vol. 505, pp. 327-34, 2014.

[40] J. Seita and L.W.Irving, "Hematopoietic Stem Cell: Self-renewal versus Differentiation." Wiley Interdiscip Rev SystBiol Med., vol. 2(6), pp. 640-653, 2010.

[41] X. Shi, Y. Zhang, J. Zheng and J. Pan, "Reactive oxygen species in cancer stem cells". Antioxid Redox Signal, vol. 16(11), pp. 1215-1228, 2012.

[42] J.E. Visvader and G.J. Lindeman, "Cancer stem cells in solid tumours: accumulating evidence and unresolved questions". Nat Rev Cancer vol. 8(10), pp. 755-768, 2008.

[43] C.E. Eyler and J.N. Rich, "Survival of the fittest: cancer stem cells in therapeutic resistance and angiogenesis. J Clin Oncol., vol. 26(17), pp. 2839-2845, 2008.

[44] A.A. Dayem, H.Y. Choi, J.H. Kim and S.G. Cho, "Role of oxidative stress in stem, cancer, and cancer stem cells". Cancers vol. 2(2), pp. 859-884, 2010.

[45] M. Diehn, R.W. Cho and N.A. Lobo, "Association of reactive oxygen species levels and radio resistance in cancer stem cells". Nature, vol. 458(7239), pp. 780-783, 2009.

[46] T. Lonergan, B. Bavister and C. Brenner, "Mitochondria in stem cells". Mitochondrion, vol. 7(5), pp. 289-296, 2007.

[47] G. Kroemer and J. Pouyssegur, "Tumor cell metabolism: cancer's Achilles' heel”. Cancer Cell, vol. 13(6), pp. 472-482, 2008.

[48] S.M. Schieke, M. Ma and L. Cao, "Mitochondrial metabolism modulates differentiation and teratoma formation capacity in mouse embryonic stem cells". J BiolChem., vol. 283, pp. 28506-12, 2008.

[49] Z. Derdak, P. Fulop and E. Sabo, "Enhanced colon tumor induction in uncoupling protein-2 deficient mice is associated with NF-kappaB activation and oxidative stress". Carcinogenesis, vol. 27(5), pp. 956-961, 2006. 
[50] D. Trachootham, J. Alexandre and P. Huang, "Targeting cancer cells by ROS-mediated mechanisms: a radical therapeutic approach". Nat Rev Drug Discov., vol. 8(7), pp. 579-591, 2009.
[51] P. D. Ray, B.-W. Huang, and Y. Tsuji, "Reactive oxygen species (ROS) homeostasis and redox regulation in cellular signaling," Cellular Signalling, vol. 24(50), pp. 981-990, 2012. 